This is an electronic reprint of the original article. This reprint may differ from the original in pagination and typographic detail.

Author(s): Salo, Markus

Title: $\quad$ Sources of Dissatisfaction: Mobile Interaction with the Real World and Other Mobile Internet Applications

Year: $\quad 2013$

Version:

Please cite the original version:

Salo, M. (2013). Sources of Dissatisfaction: Mobile Interaction with the Real World and Other Mobile Internet Applications. In R. H. Sprague (Ed.), Proceedings of the 46th Hawaii International Conference on System Sciences (HICSS) (pp. 1113-1122). IEEE Computer Society. Proceedings of the Annual Hawaii International Conference on System Sciences. https://doi.org/10.1109/HICSS.2013.493

All material supplied via JYX is protected by copyright and other intellectual property rights, and duplication or sale of all or part of any of the repository collections is not permitted, except that material may be duplicated by you for your research use or educational purposes in electronic or print form. You must obtain permission for any other use. Electronic or print copies may not be offered, whether for sale or otherwise to anyone who is not an authorised user. 


\title{
Sources of Dissatisfaction: Mobile Interaction with the Real World and Other Mobile Internet Applications
}

\author{
Markus Salo \\ University of Jyväskylä, Department of Computer Science and Information Systems \\ Mattilanniemi 2, 40100 Jyväskylä, Finland \\ markus.t.salo[at]jyu.fi
}

\begin{abstract}
Current consumer-level mobile Internet applications involve many potential sources of dissatisfaction. Studying these sources is necessary, especially for newer application areas such as mobile interaction with the real world. This study combined previous studies to form a categorization framework of dissatisfaction sources and gathered qualitative data using critical incident technique and overall evaluation questionnaire to describe the sources in detail. This article proposes insights into characteristics, temporality, and combinations of sources.
\end{abstract}

\section{Introduction}

Mobile Internet applications have become a popular phenomenon among research and business communities, but a deeper understanding of consumer aspects is needed. Current consumer-level mobile applications that are available to smart phone users via application marketplaces are still in their infancy and involve many sources of user dissatisfaction. To understand how mobile applications and human behavior go together, there is a need to focus on the sources on a contextual rather than abstract level.

The context of this study is twofold: it consists of mobile Internet applications in general with a special emphasis on mobile interaction with the real world (MIRW). Although mobile Internet applications have become familiar to nearly every owner of a smart phone with an Internet connection, MIRW represents an area of new consumer-level applications that allow users to perform physical interactions with their mobile phones in relation to the surrounding environment. In this study, the considered MIRW applications are mainly based on mobile device cameras as camerabased applications represent a large share of consumerlevel MIRW applications. Using camera-based applications, users are able to view their surroundings through the camera of the phone and receive, create, or share information by interacting physically with nearby objects or places. Example applications include seethrough displays [3][7] such as Layar and Junaio and recognition-based applications such as Google Goggles and barcode or $Q R$ code scanners. Additionally, MIRW can be also based on RFID, NFC, sensors, beams, smart objects, Bluetooth, and infrared [17].

Researchers have highlighted the importance of understanding (dis)satisfaction related to products and services: (dis)satisfaction has an effect on competitive advantage, customer loyalty, and economic success [5][21]. More specifically, researchers have found that dissatisfaction affects product- or service-related use continuance, switching behavior, complaints, and negative word-of-mouth [1][2]. Therefore, understanding dissatisfaction can help mobile application providers to maintain success by preventing user losses and negative reputation.

The objectives of this study are to 1) identify the sources of dissatisfaction that are relevant in the context of MIRW and other mobile Internet applications and 2) understand why and how such sources affect dissatisfaction. This article carries out the first objective by combining context-specific sources of dissatisfaction from prior studies. For the second objective, this article presents empirical evidence by analyzing actual experiences of 181 users and overall evaluations by 126 respondents.

\section{Dissatisfaction}

Numerous research efforts have concentrated on product or service (dis)satisfaction in recent decades. However, it seems there is no consensus on the concept or the sources of (dis)satisfaction. Some researchers define dissatisfaction as the exact opposite of satisfaction, but others understand both concepts as separate dimensions [8]. It is easy to find studies that measure the effects of various factors on the onedimensional dissatisfaction-satisfaction continuum. However, there are other widely cited studies (e.g., $[13][14])$ stating that satisfaction and dissatisfaction 
are affected by different sources. For example, there are factors that "increase dissatisfaction when absent but do not increase satisfaction when present" [21]. Thus, this study argues that dissatisfaction and satisfaction are not extremes of one continuum, so the two must be distinguished. In this article, dissatisfaction is defined as a subjective state of displeasure that originates from a negative perception of (an aspect of) a product or a service.

On an abstract level, there are three sources of dissatisfaction: external (other-oriented), internal (consumer mistakes), and situational [14]. Even though external sources are often highlighted, researchers should not overlook internal and situational sources. A mainly external source, product quality, has been found to be the most important determinant of dissatisfaction [9]. On a more specific level, (dis)satisfaction depends upon the context of the researched phenomenon, and researchers should adopt context-relevant definitions and measures [8][21]. Because this article studies mobile application context, the author chose to specify the abstract sources of [14] by applying the contextspecific studies presented as follows.

With self-service technologies and their users, a qualitative CIT study has concluded that the contextspecific sources of dissatisfaction are technology failure, poor design, process failure, and consumerdriven failure [13]. By researching both utilitarian and hedonic mobile information services with test users, a variance study (as described by [22]) has found quality aspects of connection, content, interaction, and context as antecedents of (dis)satisfaction [11]. By investigating mobile music services with test users, another variance study concludes that (dis)satisfaction is affected by quality aspects of content, context, device, connection, privacy, and perceived value, but not interaction quality or customer service - in contrast to prior literature [23]. Considering value-added mobile services and their actual users, yet another variance study found that content, navigation and visual design, management and customer service, system reliability and connection quality, and perceived value impact (dis)satisfaction [12]. Another variance study of mobile RFID applications with pilot users found that (dis)satisfaction is affected by content, interaction, customer service, and tag recognition, but not by connection quality [16]. With actual users of MIRW, our preliminary CIT study found that all of the previously presented sources are relevant, except for privacy [18]. Additionally, we found the need for adding a category labeled compatibility.

Most of the considered studies are variance studies that investigate the phenomenon by quantitative methods and structural equation modeling from the aspects of potential users or test users. Because variance studies typically answer such questions as "what are the antecedents or consequences of the issue" [22], little do they reveal about how and why certain sources affect dissatisfaction. For example, the considered variance studies make little or no contribution to the characteristics, temporality, or potential combinations of the sources. Thus, there is a need to research the relatively unexplored how and why questions. One proper way to answer such questions is to use qualitative methods based on actual user experiences. By following one of the suggested approaches of [15], the author formed a framework for a qualitative study based on prior studies. The considered studies and proposed sources of (dis)satisfaction are combined in Table 1. The description of each source is adapted and modified from our pre-study [18] and presented in Appendix A.

\section{Method}

The empirical part aims to answer "how and why" questions about the sources of dissatisfaction using two qualitative sub-studies: a critical incident technique (CIT) study and an overall evaluation questionnaire. The same framework was used for both sub-studies.

\subsection{Sub-study 1: CIT study on dissatisfying experiences}

Method. CIT is one of the three primary methods of studying (dis)satisfaction [21]. It is suitable especially in service research [10], where it has been applied in various contexts [4][6][13]. Simply put, CIT is a method to "collect, content analyze, and classify observations of human behavior" [10]. The focus of the method is on gaining a deep understanding of the investigated phenomenon rather than providing generalizable results [13]. It is useful in describing a relatively new phenomenon [10] such as MIRW.

In practice, researchers can ask respondents to describe their most positive and negative experiences with a certain service or technology. An advantage of CIT is that such experiences are easy to remember and describe, and the method unveils what is meaningful for the respondents. However, [10][21] have listed main weaknesses of the technique. First, CIT captures only the extremes of dissatisfying experiences. Second, there is a possibility of recall bias. Third, the analysis of a qualitative data set always involves a degree of subjectivity. In this study, these weaknesses were addressed by investigating the same phenomenon with an overall evaluation sub-study, providing the respondents with clear instructions and measuring interrater reliability. 


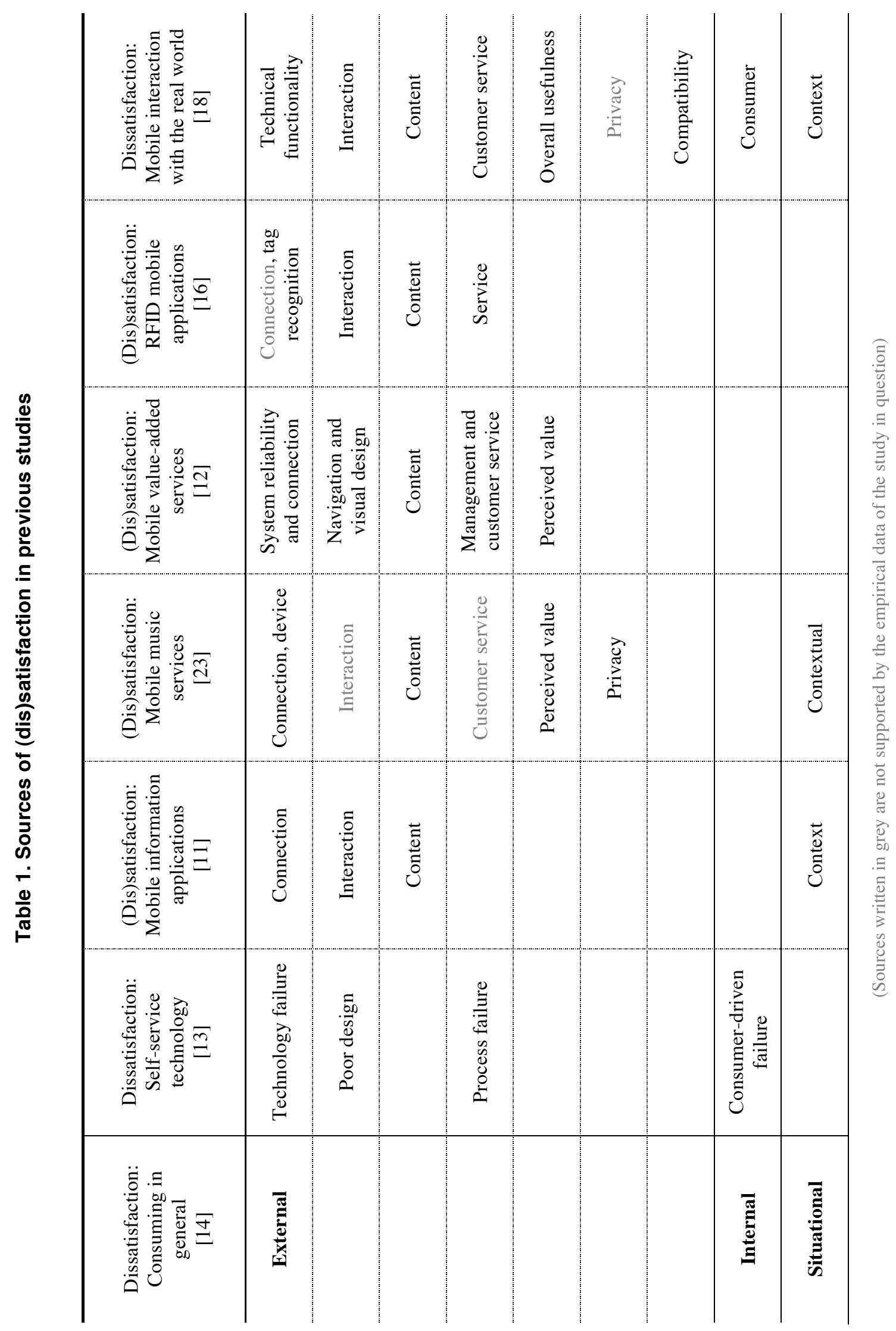


Study design. The study primarily followed a checklist for CIT content analytic studies by [10]. We delivered web forms for international MIRW application users during the spring of 2011, and for Finnish mobile Internet users during the spring of 2012. The first data set was also used in our earlier study, which investigated dissatisfaction in MIRW applications [18]. However, because this study examines both MIRW and other mobile Internet applications using two substudies, it is reasonable to include the same data set as a part of the data of this study.

Respondents were required to have previous practical experience with the applications. Respondents were instructed to describe their most dissatisfying experience in their own words in as much detail as possible by reporting the overall situation, the aspects that caused dissatisfaction, and their feelings. To motivate respondents, prizes were raffled. Before conducting the sub-study, fellow researchers and pilot respondents provided comments about the web form.

Analysis. In total, there were 181 respondents who each provided a sufficient description of a dissatisfying experience. As [10] suggests, an inclusion criterion for critical incidents was defined: response was considered valid when it described a dissatisfying experience of any investigated mobile application with a sufficient level of detail regarding the source(s) of dissatisfaction of that particular experience. The data showed that some of the descriptions reflected more than just one source, so each description was coded in one or more source categories. Hence, the analysis of 181 descriptions resulted in a total of 226 codings.

To enhance objectivity, interrater and intrarater reliability were measured as suggested by [10] and [19]. Two researchers, blind to each other's codings, conducted the analysis of the spring 2011 data (65 respondents). Consensus estimate of interrater reliability was measured by dividing the amount of identically placed codings by the total number of codings, and it resulted in a percent-agreement figure of $84.6 \%$. In service research CIT studies, analysis is considered to be reliable when the percentage is $80 \%$ or higher [10]. Additionally, the other researcher analyzed the spring 2011 data for the second time after six months to measure intrarater reliability. The consensus estimate of intrarater reliability was $96.2 \%$.

Respondents. The group of 181 respondents consisted mainly of technologically oriented persons, based on their rather high agreement with statements about technology use. A majority (76.8\%) of them were male, under the age of 35 years $(79.6 \%)$, Finnish (76.2\%), and had completed a bachelor's degree or higher $(55.2 \%)$. However, they ranged in age from 16 to 65 years, and there were 17 nationalities represented in the sample. The responses are related to a total of 34 different applications. The group of respondents fits well with this study as the aim is to describe the relevant sources rather than to generalize the results. However, limitations are discussed in section 6 .

\subsection{Sub-study 2: Overall evaluation questionnaire}

Method. Because the CIT study concentrates on extreme experiences, it might not cover all aspects of dissatisfaction sources and their complete descriptions. Thus, overall evaluation of dissatisfaction sources was examined by constructing a qualitative questionnaire for respondents familiar with the framework of this study.

Design. The sub-study was affiliated with a bachelor-level course of information systems science about business in e-society. The course included an orientation lecture about mobile applications, MIRW, and sources of dissatisfaction, and each student had access to the lecture material afterwards. In a home exam, students were asked to prioritize the three most crucial sources of dissatisfaction from the presented ones. Additionally, each student had to include a halfpage justification for his or her answers. The students were motivated to write detailed and reasoned responses by giving each valid response a set of points that affected the final grade.

Analysis. The analysis was carried out with content analysis by following the instructions of [20]. The author organized the data by the sources of dissatisfaction and examined each source separately. The purpose was to prepare the data for reporting tendencies related to the sources, although exceptions and minor issues were also noted.

Respondents. Similar to the sub-study 1, a majority of the students who completed the course were male $(71.3 \%)$ and under the age of 35 years (94.6\%). The respondents ranged in age from 19 to 48 years. All of them were Finnish, and most of them were in a bachelor of information systems science program. More than half of the respondents indicated that they had actual experience on the applications they discussed in their responses. It is possible that some persons participated in both sub-studies. Again, limitations are discussed in section 6.

\section{Results}

The data sets of the sub-studies reflected each of the proposed sources of dissatisfaction (Tables 2 and 3). The most frequent sources were technical functionality, content, and interaction. The sources are described as follows. 
Table 2. Results of sub-study 1: CIT study on dissatisfying experiences

\begin{tabular}{|c|c|c|c|c|}
\hline \multicolumn{2}{|c|}{ Source of dissatisfaction } & \multirow{2}{*}{$\begin{array}{c}\text { MIRW } \\
\text { applications: } \\
102 \text { descriptions } \\
\text { Codings (\% of } \\
\text { descriptions) } \\
\end{array}$} & \multirow{2}{*}{$\begin{array}{l}\text { Other mobile } \\
\text { applications: } \\
79 \text { descriptions } \\
\text { Codings (\% of } \\
\text { descriptions) }\end{array}$} & \multirow{3}{*}{$\begin{array}{c}\text { Total: } \\
181 \text { descriptions } \\
\begin{array}{c}\text { Codings (\% of total } \\
\text { descriptions) }\end{array} \\
94(51.9 \%)\end{array}$} \\
\hline \multirow{8}{*}{ External } & & & & \\
\hline & $\begin{array}{c}\text { Technical } \\
\text { functionality }\end{array}$ & $57(55.9 \%)$ & $37(46.8 \%)$ & \\
\hline & Content & $46(45.1 \%)$ & $8(10.1 \%)$ & $54(29.8 \%)$ \\
\hline & Interaction & $9(8.8 \%)$ & $22(27.8 \%)$ & $31(17.1 \%)$ \\
\hline & Privacy & $0(0 \%)$ & $10(12.7 \%)$ & $10(5.5 \%)$ \\
\hline & Compatibility & $5(4.9 \%)$ & $4(5.1 \%)$ & $9(5.0 \%)$ \\
\hline & Overall usefulness & $6(5.9 \%)$ & $1(1.3 \%)$ & $7(3.9 \%)$ \\
\hline & Customer service & $3(2.9 \%)$ & $0(0 \%)$ & $3(1.7 \%)$ \\
\hline Internal & Consumer & $3(2.9 \%)$ & $5(6.3 \%)$ & $8(4.4 \%)$ \\
\hline \multirow[t]{2}{*}{ Situational } & Context & $9(8.8 \%)$ & $1(1.3 \%)$ & $10(5.5 \%)$ \\
\hline & Total codings & 138 & 88 & 226 \\
\hline
\end{tabular}

Table 3. Results of sub-study 2: Overall evaluation questionnaire

\begin{tabular}{|c|c|c|c|c|}
\hline \multicolumn{2}{|c|}{ Source of dissatisfaction } & $\begin{array}{c}\text { MIRW } \\
\text { applications: } \\
63 \text { respondents } \\
\text { Respondents who } \\
\text { prioritized in top } 3 \\
\text { (\% of total } \\
\text { respondents) }\end{array}$ & $\begin{array}{c}\text { Other mobile } \\
\text { applications: } \\
63 \text { respondents } \\
\text { Respondents who } \\
\text { prioritized in top } 3 \\
\text { (\% of total } \\
\text { respondents) }\end{array}$ & $\begin{array}{c}\text { Total: } \\
126 \text { respondents }\end{array}$ \\
\hline \multirow[t]{7}{*}{ External } & $\begin{array}{c}\text { Technical } \\
\text { functionality }\end{array}$ & $53(84.1 \%)$ & $52(82.5 \%)$ & $105(83.3 \%)$ \\
\hline & Content & $39(61.9 \%)$ & $30(47.6 \%)$ & $69(54.8 \%)$ \\
\hline & Interaction & $40(63.5 \%)$ & $38(60.3 \%)$ & $78(61.9 \%)$ \\
\hline & Privacy & $7(11.1 \%)$ & $5(7.9 \%)$ & $12(9.5 \%)$ \\
\hline & Compatibility & $9(14.3 \%)$ & $16(25.4 \%)$ & $25(19.8 \%)$ \\
\hline & Overall usefulness & $26(41.3 \%)$ & $22(34.9 \%)$ & $48(38.1 \%)$ \\
\hline & Customer service & $1(1.6 \%)$ & $7(11.1 \%)$ & $8(6.3 \%)$ \\
\hline Internal & Consumer & $9(14.3 \%)$ & $6(9.5 \%)$ & $15(11.9 \%)$ \\
\hline Situational & Context & $5(7.9 \%)$ & $13(20.6 \%)$ & $18(14.3 \%)$ \\
\hline
\end{tabular}




\subsection{Technical functionality}

Sub-study 1. According to the CIT data, approximately half $(51.9 \%)$ of the dissatisfying experiences include issues related to technical functionality during the actual use situation. There are both application (or software) and device (or hardware) issues. Considering application issues, users of both MIRW and other applications have experienced dissatisfaction due to common issues such as bugs, errors, crashes, and slowness. These basic failures are common with MIRW, while the users of other applications stress more sophisticated failures related to time-criticality and service reliability. For example, one respondent trusted that she could handle buyerseller communication with a mobile application, but she reported a failed trade and a loss of 50-100 Euros in savings due to the errors of the application.

For device issues, MIRW users especially had problems with features related to GPS, compass, camera, and network connection. Naturally, users of other applications did not stress such features. Some of the device-related descriptions are linked to context as technical functionality may depend on surrounding conditions. For example, tall buildings may prevent GPS from finding accurate locations, and rural areas make fast network connections challenging.

Sub-study 2. According to the overall evaluation data, a majority of the respondents $(83.3 \%)$ mentioned technical functionality as one the three most crucial sources. Respondent perceptions are mainly in line with the CIT data. The respondents emphasized that users in general are impatient in time-critical mobile situations, and they get fed up with applications immediately if the applications do not perform well. Failures thus result commonly in discontinued use, so the very first experiences are crucial.

\subsection{Content}

Sub-study 1. Regarding MIRW applications, content (reflected in 46/102 descriptions) is a major source of dissatisfaction. In the CIT data, the main reason for this is the lack of content with added value: At times, content was not fresh, local, unique, or unlimited. In some of the worst cases, there was no content at all; it was misplaced or flawed; or the application was overcrowded with content. Some users reported that many content issues appear due to the infancy of MIRW applications. Additionally, a set of dissatisfying experiences results from a combination of content and technical functionality. In some cases, content was misplaced or flawed due to wrong GPS coordinates or incorrect image recognition.
With other applications, there were fewer contentrelated dissatisfying experiences (9/79), and they were mainly related to special occasions. For example, one respondent reported that a user-generated flood of irrelevant content made him frustrated.

Sub-study 2. In the overall evaluation data, approximately half $(54.8 \%)$ of the respondents mentioned content as one of the three primary sources of dissatisfaction. The reasoning for the importance of content is in line with the CIT data. The respondents tend to seek diverse but trustworthy content that ismost importantly-filtered to match user preferences.

\subsection{Interaction}

Sub-study 1. The CIT data shows that dissatisfaction may arise from complexity and inconvenience of use. In such cases, dissatisfaction relates to interaction that is not intuitive, responsive, easy, pleasant, understandable, or properly finalized. Additionally, with applications that are more mature and used more frequently, changes in interaction and design may cause feelings of confusion and lack of control. There are relatively few MIRW-related descriptions that reflect interaction (9/102) compared to other applications $(22 / 79)$.

Sub-study 2. Based on the overall evaluation data, more than half of the respondents $(61.1 \%)$ consider interaction as one of the primary sources, and they tend to perceive many applications too complicated to use on the go. They expect that new technology, including MIRW, should be less complex to use than other alternatives. In general, respondents state that users do not prefer learning new manners to use applications unless the instructions are excellent or the applications provide very positive experiences.

\subsection{Privacy}

Sub-study 1. In the CIT data, there are no dissatisfying experiences that were caused by privacy related to MIRW applications. However, with other applications, ten experiences relate to privacy concerns of social networking applications. Such experiences result from lack of control over personal information, changes in privacy settings, and perceived insecurity. For example, one respondent mentioned that the application provider does not indicate clearly how it uses personal information.

Sub-study 2. A small number of respondents (9.5\%) prioritized privacy among the most crucial sources of dissatisfaction for all application types. The overall evaluation data covers the same themes as the CIT data: lack of control, privacy settings, and insecurity. 


\subsection{Compatibility}

Sub-study 1. According to the CIT data, a handful of critical experiences $(5.0 \%)$ reflect compatibility. Some of the MIRW applications had caused dissatisfaction as they were difficult or impossible to integrate with other applications or data sources. In a few cases, the desired application was not available for certain mobile platforms, or they malfunctioned after an operation system update. With other applications, dissatisfaction appeared due to unsupported file types and inability to export personal exercise data, as well as poor compatibility with other applications and exercise hardware.

Sub-study 2. In the overall evaluation data, $19.8 \%$ of the respondents highlighted compatibility. The respondents stressed compatibility between applications as for data exporting and importing. For example, some respondents stated that users are willing to synchronize personal data, such as calendar events, and share information with friends by exporting data from one application to another.

\subsection{Overall usefulness}

Sub-study 1. In the CIT data, overall usefulness is related nearly only to MIRW applications (6/7). Most of these cases are first experiences and tests, in which the applications do not live up to expectations regarding overall usefulness combined with technical functionality or content. In consequence, users perceive such applications as useless. However, the small number of codings in this category might be explained by the assumption that single critical experiences rarely reflect abstract issues such as overall usefulness.

Sub-study 2. With the overall evaluation data, $38.1 \%$ of the respondents prioritized overall usefulness in the top three sources of dissatisfaction. If a mobile application has no relative advantage over other alternatives, it may cause dissatisfaction and discontinued use. Some of the respondents state that MIRW applications should provide long-lasting utility in addition to wow-feelings. Sometimes such usefulness is linked with content. Overall usefulness may be judged by comparing benefits and sacrifices, which is a subjective process. For example, in contrast to many others, one respondent argued that he preferred the traditional way of typing text into a search box more than taking a picture to start a web search.

\subsection{Customer service}

Sub-study 1. In the CIT data, there are only three experiences that reflect customer service. All of them are related to MIRW applications that provide poor documentation or instructions for creating content, integrating databases, or using the applications.

Sub-study 2. In the overall evaluations, a small number of respondents $(6.3 \%)$ highlight the effects of customer service, and only one was related to MIRW applications. According to the respondents, it is not necessarily the application providers that should provide life cycle support but also device manufacturers, sellers, and data service providers.

\subsection{Consumer}

Sub-study 1. There are eight experiences in the CIT data that reflect consumer-driven failure. In some cases, users just do not know how to use the application (such as how to remove personal pictures from their account). There is also the possibility of human error. In one case, an image search application could not function because the respondent shook the camera while taking a picture. Some of the experiences consist of interaction-related issues such as clicking the wrong button continuously. There were various other data errors: one user forgot a password and another made a mistake due to inebriation. The relatively small number of reported consumer failures might be explained by the assumption that consumers are usually unwilling to take the blame for failure.

Sub-study 2. In the overall evaluation data, $12.7 \%$ of the respondents prioritized consumer failures. The issues are experience, skills, and knowledge. A rather common argument was that even though a mobile application is designed properly, some users find it difficult to use. A few respondents speculated that in such cases users might blame the application instead of the actual source of error-the user.

\subsection{Context}

Sub-study 1. In the CIT data, there were nine negative experiences with MIRW applications, but only one negative experience with other applications was caused by context-related factors. The users reported situations where the surrounding environment, such as poor lighting conditions, caused a failure in either the use of a device or the technical functionality of the Internet connection, camera, compass, or other features. These issues are more related to place than time. One user noted an issue of social acceptance: the user was "harassed by shop staff for taking photos of barcodes".

Sub-study 2. According to the overall evaluation data, $14.3 \%$ of the respondents mentioned context as one of the primary sources. The respondents reported issues similar to the CIT data. 


\section{Discussion and implications}

This study contributes to the knowledge of sources of dissatisfaction in the context of mobile Internet applications. The data sets included responses from both actual and potential users, different from many earlier research efforts that have concentrated on only one or the other. The findings suggest that all of the proposed sources are relevant in the context of mobile applications. Technical functionality, content, and interaction were the most frequently reported sources. Prior research has found that these sources are significant determinants of (dis)satisfaction [11][12][13], although the relationship between interaction and (dis)satisfaction has not always been supported [23]. Application providers could use the framework of this study to form a checklist to improve their applications and compare dissatisfaction over time. More importantly, the findings of this study contribute to the characteristics, temporality, and combinations of the sources as follows.

Characteristics of each source. Each source of dissatisfaction actualizes with different characteristics, which depend on the application area, its maturity, and user involvement. This proposition is illustrated by the following two examples. First, failures in technical functionality and content are frequent and cogent with MIRW as the application area is still under construction and the applications typically require several technical features to function. This is supported by the CIT data as a great number of MIRW users reported a diverse repertoire of major teething problems such as crashes or having no relevant content at all. Yet users of other applications reported only mediocre technical and content failures that were often related to special occasions. Second, privacy concerns become evident by requiring user involvement. With social networking applications, it is the high user involvement in the form of sharing personal information that causes privacy concerns. With MIRW applications, privacy concerns have not yet reached actual usage, but they are expected to do so in the future, when the applications implement social or personal features. By considering application type, its maturity and required user involvement, application providers could reach specific insights that go beyond the abstract sources of dissatisfaction.

Proposition 1. Application area, its maturity, and required user involvement define the characteristics of each source of dissatisfaction.

Temporality. Most of the referred studies investigate (dis)satisfaction with a more or less static model of the phenomenon. This article proposes that dissatisfaction is a dynamic phenomenon, and it should be investigated by taking the temporal dimension into account. It was the CIT study that especially helped the author to understand temporal differences, which would have been difficult to figure with a typical variance study that does not highlight temporality [22].

As the results suggest, the three major sources (technical functionality, content, and interaction) cause dissatisfaction in a rather narrow time frame: during the actual use situation. With these sources, dissatisfaction typically occurs due to punctuated failures that users come across during use. However, with other sources, the relevant time frame can be wider. Dissatisfaction may appear before the actual use situation when, for example, users are not able to even download an application if it is not compatible with their version of an operating system. Privacy concerns, as another example, sometimes appear after use, as well as poor customer service, which may cause dissatisfaction in any phase of the before use-after use continuum. In spite of the finding that most sources of dissatisfaction occur during the actual use situation, application providers should learn to understand the temporal dimension of dissatisfaction and consider all relevant phases when evaluating and developing their applications.

Proposition 2. Different sources of dissatisfaction may appear in different temporal phase(s).

Combinations of sources. Most of the referenced studies do not include discussion about combinations of factors or categories determining (dis)satisfaction. But in this study, a number of critical incidents clearly reflect more than one source at a time. Thus, this article proposes that sometimes dissatisfaction occurs due to combinations of sources.

By investigating such combinations further, the author noted that many of the combined sources seem to have causal relationships and temporal order: something happens at first, causing something else to happen. For example, with MIRW applications, some context-related troubles lead to poor technical functionality. In a similar manner, poor technical functionality can cause content failures. The sources could also form combination chains that affect dissatisfaction: Context may cause failure in technical functionality, which in turn may cause content failure. However, further research is necessary to confirm such specific causal relationships.

Proposition 3. Dissatisfaction may occur due to combinations of sources, which appear in temporal order and have causal relationships. 


\section{Limitations and future research topics}

There are certain limitations of this study. First, the groups of respondents in both sub-studies were relatively specific and small. Thus, one should not draw any additional conclusions about the frequency of the sources. Second, CIT is a method that relies on respondents' memory, so there might be recall bias. Third, the overall evaluation sub-study was based on working from a predefined framework. So those respondents were given a set of options to choose from, and some of those respondents might not have familiarized themselves with the framework in full detail. However, these limitations do not critically affect this study as the aim of the empirical part was to answer how and why questions.

In the future, research efforts could inspect the propositions of this study in more detail. As dissatisfaction affects use continuance, complaints, and word-of-mouth [1][2], such consequences could be studied in the mobile context using mixed methods similar to [13]. Additionally, a quantitative study of overall evaluation with a representative group of respondents would reveal useful information about frequencies of the sources as well as open possibilities for conducting statistical comparisons between application categories.

\section{References}

[1] J-H. Ahn, S-P. Han, and Y-S. Lee, "Customer Churn Analysis: Churn Determinants and Mediation Effects of Partial Defection in the Korean Mobile Telecommunications Service Industry", Telecommunications Policy, Vol. 30, No. 10-11, 2006, pp. 552-568.

[2] A. Bhattacherjee, "Understanding Information Systems Continuance: An Expectation-Confirmation Model", MIS Quarterly, Vol. 25, No. 3, 2001, pp. 351-370.

[3] E. Bier, M. Stone, K. Pier, W. Buxton, and T. DeRose, "Toolglass and Magic Lenses: The See-Through Interface", 20th Annual Conference on Computer Graphics and Interactive Techniques (SIGGRAPH), 1993, pp. 73-80.

[4] M.J. Bitner, B.H. Booms, and M.S. Tetreault, "The Service Encounter: Diagnosing Favorable and Unfavorable Incidents", The Journal of Marketing, Vol. 54, No. 1, 1990, pp. 71-84.

[5] Z. Deng, Y. Lu, K.K. Wei, and J. Zhang, "Understanding Customer Satisfaction and Loyalty: An Empirical Study of Mobile Instant Messages in China", International Journal of Information Management, Vol. 30, No. 4, 2010, pp. 289-300.

[6] B. Edvardsson, "Causes of Customer DissatisfactionStudies of Public Transport by the Critical-Incident Method", Managing Service Quality, Vol. 8, No. 3, 1998, pp. 189-197.

[7] P. Fröhlich, A. Oulasvirta, M. Baldauf, and A. Nurminen, "On the Move, Wirelessly Connected to the World", Communications of ACM, Vol. 54, No. 1, 2011, pp. 132-138.
[8] J.L. Giese, and J.A. Cote, "Defining Consumer Satisfaction. Academy of Marketing Science Review", No. 1, 2000.

[9] M.M.H. Goode, F. Davies, L. Moutinho, and A. Jamal, "Determining Customer Satisfaction from Mobile Phones: A Neural Network Approach", Journal of Marketing Management, Vol. 21, No. 7-8, 2005, pp. 755-778.

[10] D.D. Gremler, "The Critical Incident Technique in Service Research", Journal of Service Research, Vol. 7, No. 1, 2004, pp. 65-89.

[11] T. Koivumäki, A. Ristola, and M. Kesti, "The Effects of Information Quality of Mobile Information Services on User Satisfaction and Service Acceptance-Empirical Evidence from Finland", Behaviour \& Information Technology, Vol. 27, No. 5, 2008, pp. 375-385.

[12] Y.F. Kuo, C.H. Wu, and W.J. Deng, "The Relationships among Service Quality, Perceived Value, Customer Satisfaction, and Post-Purchase Intention in Mobile ValueAdded Services", Computers in Human Behavior, Vol. 25, No. 4, 2009, pp. 887-896.

[13] M.L. Meuter, A.L. Ostrom, R.I. Roundtree, and M.J. Bitner, "Self-Service Technologies: Understanding Customer Satisfaction with Technology-Based Service Encounters", Journal of Marketing, Vol. 64, No. 3, 2000, pp. 50-64.

[14] R. Oliver, "Effect of Expectation and Disconfirmation on Postexposure Product Evaluations: An Alternative Interpretation", Journal of Applied Psychology, Vol. 62, No. 4, 1977, pp. 480-486.

[15] G. Paré, "Investigating Information Systems with Positivist Case Study Research", Communications of the Association for Information Systems, Vol. 13, No. 1, 2004, pp. 233-264.

[16] Y-J. Park, P-S. Heo, and M-H. Rim, "Measurement of a Customer Satisfaction Index for Improvement of Mobile RFID Services in Korea", ETRI Journal, Vol. 30, No. 5, 2008, pp. 634-643.

[17] E. Rukzio, "Physical Mobile Interactions: Mobile Devices as Pervasive Mediators for Interactions with the Real World", Ph.D. Thesis, Faculty for Mathematics, Computer Science and Statistics, University of Munich, 2007.

[18] M. Salo, and T. Olsson, "Consumer dissatisfaction of mobile interaction with the real world", IADIS International Conference on e-Society, 2012, pp. 399-408.

[19] S.E. Stemler, "A Comparison of Consensus, Consistency, and Measurement Approaches to Estimating Interrater Reliability", Practical Assessment, Research \& Evaluation, Vol. 9, No. 4, 2004.

[20] J. Tuomi, and A. Sarajärvi, Laadullinen tutkimus ja sisällönanalyysi, Tammi, Helsinki, 2002.

[21] S.L. Vargo, K. Nagao, Y. He, and F.W. Morgan, "Satisfiers, Dissatisfiers, Criticals, and Neutrals: A Review of Their Relative Effects on Customer (Dis)Satisfaction", Academy of Marketing Science Review, Vol. 11, No. 2, 2007.

[22] A.H. Van de Ven, Engaged Scholarship: A Guide for Organizational and Social Research, Oxford University Press, New York, 2007.

[23] P.A. Vlachos, and A.P. Vrechopoulos, "Determinants of Behavioral Intentions in the Mobile Internet Services Market", Journal of Services Marketing, Vol. 22, No. 4, 2008, pp. 280-291. 


\section{Appendix A. Research framework and descriptions of dissatisfaction sources (adapted and slightly modified from [18])}

\section{Source of dissatisfaction}

External

TECHNICAL FUNCTIONALITY

INTERACTION

CONTENT

CUSTOMER SERVICE

PRIVACY

COMPATIBILITY

OVERALL USEFULNESS

Internal

CONSUMER

Situational

CONTEXT

\section{Description}

Technology is not working as intended: instability, errors, slowness of the application, connection, device, or functions (such as location-based or camera-based recognition)

Complexity of application or device use: inconvenient interaction, weaknesses in user interface and design, lack of clarity of presentation or instructions, or confusing in-app navigation

Content is not matching user needs: useless, irrelevant, non-preferred, unwanted, skewed or incomprehensible content, excessive content, or absence of content

Weaknesses in customer or developer support: lack of or delay in customer service, unhelpful service, incomplete or limited instructions and support documentation (such as FAQs)

Privacy concerns: poor or confusing treatment and protection of personal information, insecurity of the application

Lack of adaptability to earlier conditions of the user: incompatibility with earlier technology or user state of mind, incapable of being integrated with other applications or data sources

User perceives no utilitarian value: not useful, insufficient benefits compared to sacrifices, application does not harness its potential

Consumer-driven failure: negative experience caused by the user due to the user's actions or mistakes

Inability to function whenever and wherever: uncommon conditions, interference from the physical environment, other unfortunate situational events 